\title{
INFLUÊNCIA DAS PROPRIEDADES TÉRMICAS DO CONCRETO MASSA NA ANÁLISE DA TEMPERATURA EM ESTRUTURAS DE GRANDES DIMENSÕES
}

\author{
N. A. Coelho ${ }^{1}$, L. J. Pedroso ${ }^{1}$, J. H. S. Rêgo ${ }^{1}$, A. A. Nepomuceno ${ }^{1}$ \\ ${ }^{1}$ Departamento de Engenharia Civil e Ambiental, Programa de Pós-graduação em Estruturas e \\ Construção Civil da Universidade de Brasília
}

Resumo. Com o crescente desenvolvimento brasileiro nos últimos anos, grandes obras de engenharia tem sido projetadas e construídas, em particular aquelas que envolvem grandes volumes de concreto massa, como é o caso das barragens. O concreto massa, devido as suas características de grande volume e dimensões, apresenta uma elevação de temperatura considerável devido a hidratação dos grãos de cimento. Essa elevação de temperatura pode ser suficiente para causar trincas elou fissuras no concreto, as quais são capazes de provocar graves problemas. Neste artigo buscou-se estudar a geração de calor e o campo de temperatura no concreto massa através do um programa ANSYS que utiliza o método dos elementos finitos para análise do problema. O programa permite que as temperaturas sejam verificadas para diferentes idades do concreto. Assim, pode-se avaliar as temperaturas obtidas e os fatores que influenciam nos resultados em um curto período de tempo e a um baixo custo. Com o auxílio do programa é possível se verificar as temperaturas para diferentes propriedades do concreto, analisando-as em diferentes dias de concretagem. Dessa forma, foi possível constatar que as propriedades do concreto influenciam diretamente no fenômeno da evolução da temperatura.

Keywords: Temperatura, concreto massa, isotermas, análise numérica.

\section{INTRODUÇÃO}

O concreto massa é qualquer volume de concreto com dimensões grandes o suficiente que requeiram medidas a serem tomadas para superar a geração de calor de hidratação do cimento reduzindo a mudança de volume para minimizar a fissuração [1].

Muitas obras utilizam o concreto massa na sua confecção. Tais estruturas normalmente apresentam grandes dimensões e grandes volumes de concreto e tornam-se preocupantes devido à probabilidade do surgimento de patologias, tais como fissurações e trincas. Quando 
os grãos do cimento entram em contato com a água, eles se hidratam e liberam calor, conhecido como calor de hidratação.

Geração de calor no interior de um bloco de concreto gera um gradiente de temperatura em relação aos bordos externos que, se o concreto não puder se movimentar livremente, induz o surgimento de tensões de tração que, se ultrapassarem a resistência à tração do concreto, haverá fissuração e podem causar danos e a estanqueidade da estrutura [7]. Muitos estudos tem sido efetuados na literatura, a respeito do fenômeno relativo aos efeitos térmicos no concreto massa, [3, 4, 8, 9, 12].

Alguns recursos, como a utilização de softwares, podem prever e avaliar a temperatura interna no concreto massa. Este processo permite a identificação das maiores temperaturas atingidas, do tempo de evolução do calor e dos locais de temperatura crítica na estrutura. Com esses dados é possível buscar soluções para melhor adequação ao problema.

Um exemplo de software que pode ser utilizado nesses problemas é o ANSYS, uma ferramenta poderosa que utiliza o método dos elementos finitos para solução de vários problemas de engenharia, como estruturais, dinâmicos, térmicos, entre outros. O que proporciona uma análise rápida e econômica quando comparada com ensaios experimentais.

Dessa forma, neste trabalho, o programa ANSYS será utilizado como ferramenta operacional para a visualização dos efeitos térmicos, da variação de temperatura no interior de estruturas de concreto massa. Serão estudadas a variação térmica devido as mudanças dos parâmetros da equação que governa o problema do calor no interior das estruturas.

\section{PROPRIEDADES TÉRMICAS DO CONCRETO}

O estudo da massa específica, do calor específico, da condutividade e da difusividade térmica são importantes para o desenvolvimento de gradientes de temperatura, deformações térmicas e fissuração nas primeiras idades do concreto. A seguir apresentam-se suas definições resumidamente:

- Massa específica ( $\rho)$ - é a relação entre a massa de um corpo sobre o volume que esse mesmo corpo ocupa. É influenciada pelo teor de água e de finos, pelos agregados utilizados e pelo índice de vazios. Quanto menor a quantidade de água e teor de pasta na mistura maior será a massa específica.

- Condutividade térmica (k) - capacidade do material conduzir calor, é definido como a relação entre o fluxo de calor e o gradiente de temperatura. A condutividade térmica depende da composição do concreto e o aumento da umidade também eleva o seu valor [11].

- Calor Específico (c) - é um parâmetro físico que expressa à capacidade de um material armazenar calor. O calor específico corresponde à quantidade de calor necessária para elevar em um grau a temperatura de uma unidade de massa. Valores típicos do calor específico para concretos normais estão entre $0,84 \mathrm{e}$ $1,26 \mathrm{~kJ} /(\mathrm{kg} \cdot \mathrm{K})[5]$. 
- Difusividade térmica $(\boldsymbol{\delta})$ - representa a velocidade à qual a temperatura varia no interior de uma massa, sendo, portanto um índice da facilidade com a qual o concreto pode sofrer variações de temperatura. De acordo com a literatura [2] a difusividade térmica é a propriedade que expressa a capacidade de difusão de calor em todas as direções e indica a facilidade com que o concreto tolera variações de temperatura. É calculada de acordo com a equação 1.

$$
\delta=\frac{k}{c \rho}
$$

Onde:

$$
\begin{aligned}
& \mathbf{k}=\text { condutividade térmica; } \\
& \mathbf{c}=\text { calor específico; } \\
& \rho=\text { massa específica do concreto. }
\end{aligned}
$$

Em estruturas de concreto massa o efeito da hidratação do cimento produz uma reação exotérmica com uma elevação significativa da temperatura desse material. Além do calor de hidratação existem outros fenômenos térmicos que atuam diretamente nessas construções: a radiação solar, a condução e a convecção. Uma estrutura de concreto massa gera calor nos primeiros tempos de sua existência, conduz calor através de sua massa; recebe, emite e reflete calor através de suas faces e, após certo tempo, atinge sua temperatura de equilíbrio.

\section{FUNDAMENTOS TEÓRICOS}

Um dos principais objetivos da avaliação dos efeitos térmicos é determinar o campo de temperatura em um meio resultante das condições impostas em suas fronteiras. Ou seja, deseja-se conhecer a distribuição de temperaturas, que representa como a temperatura varia com a posição no meio. Uma vez conhecida essa distribuição, o fluxo de calor por condução em qualquer ponto do meio ou na sua superfície pode ser determinado através da lei de Fourier [6].

Considera-se um meio homogêneo, e a distribuição de temperaturas $T(x, y, z)$ representada em coordenadas cartesianas. A equação geral da transmissão de calor é avaliado pelo fluxo de calor que passa através desse corpo, como é mostrado na figura 2. 


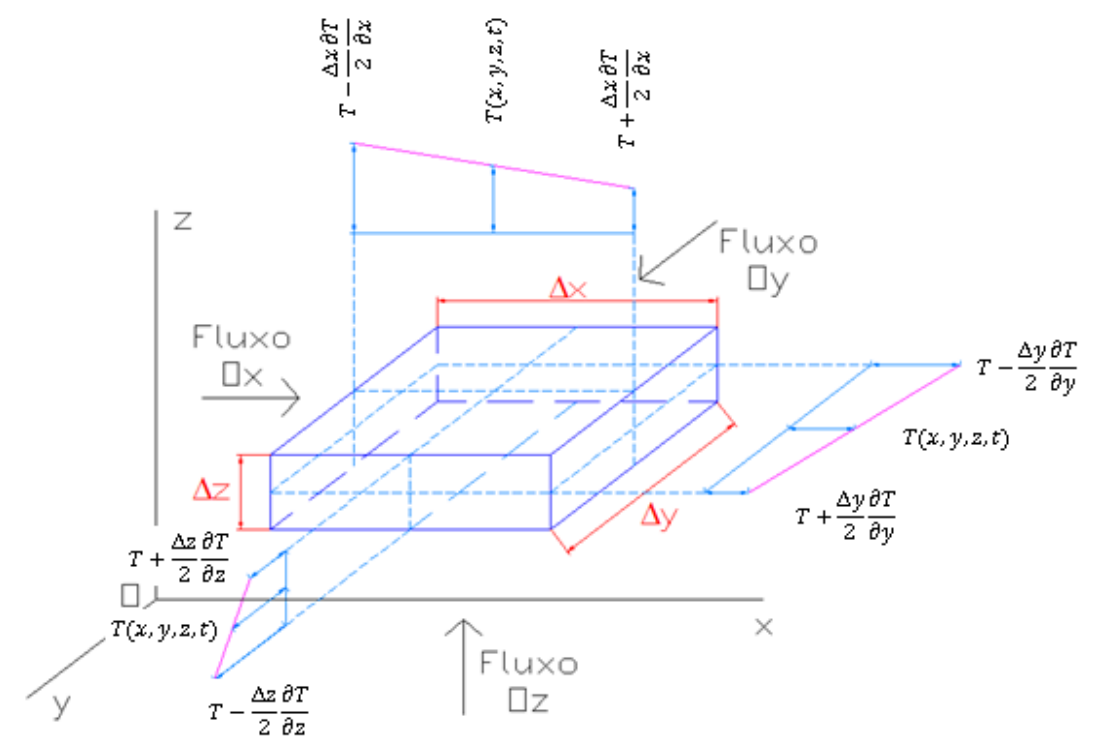

Figura 1. Corpo submetido a um fluxo de calor.

Seja $c$ o calor especifico do material, $k$ a condutividade térmica, $\rho$ sua massa especifica, $(\Delta x \Delta y \Delta z)$ o elemento de volume e $\left(\frac{d T}{d x}\right)$ o gradiente térmico na direção $\mathrm{x}$. $\mathrm{O}$ elemento de volume sofrerá, na unidade de tempo, uma elevação de temperatura em função do tempo, $\left(\frac{d T}{d t}\right)$ e chega-se a equação de Fourier mostrada em (2) e (3):

$$
\begin{gathered}
k \Delta x \Delta y \Delta z\left(\frac{\partial^{2} T}{\partial x^{2}}+\frac{\partial^{2} T}{\partial y^{2}}+\frac{\partial^{2} T}{\partial z^{2}}\right)=c \rho \Delta x \Delta y \Delta z \frac{\partial T}{\partial t} \\
k \nabla^{2} T=c \rho \frac{\partial T}{\partial t}
\end{gathered}
$$

Se o corpo desenvolver calor, como é o caso do concreto, há a influência de um outro termo na Equação de Fourier que é a representação da quantidade de calor desenvolvido, dado por $\dot{q}$. Logo tem-se a equação 4 :

$$
k \nabla^{2} T+\dot{q}=c \rho \frac{\partial T}{\partial t}
$$

Assim, a Equação Geral da Condução de Calor, considerando a Equação de Fourier e a geração de calor interno, (calor de hidratação), é dada pela da equação 5:

$$
\boldsymbol{\nabla}^{2} T+\frac{\dot{q}}{k}=\frac{c \rho}{k} \frac{\partial T}{\partial t}
$$

A equação 5 implica dizer que em qualquer ponto do meio, a taxa líquida de transferência de energia por condução para o interior de um volume unitário somada a taxa volumétrica de geração de energia térmica deve ser igual à taxa de variação de energia térmica acumulada no interior desse volume [6]. 
Para a resolução da Equação de Fourier, descrita em (5), é necessário considerar o calor de hidratação, $\dot{q}$, para o caso do concreto. O estudo mais recente, sobre a barragem de Três Gargantas na China, realizado por [10] afirma que o calor desenvolvido pela hidratação do cimento pode ser representado pela equação (6), obtida por meio de dados experimentais levando em consideração a temperatura adiabática em diferentes idades do concreto.

$$
\dot{q}=q_{i}\left(1-e^{\alpha t_{i}^{\beta}}\right)
$$

Em que:

$\dot{q}=$ calor de hidratação;

$q_{i}, \propto, \beta=$ constantes obtidas experimentalmente, depende da composição do cimento; sendo que $q_{i}$ é o calor inicial do concreto.

\section{RESULTADOS OBTIDOS COM O AUXÍLIO DO PROGRAMA ANSYS}

\subsection{Análise dos parâmetros característicos presentes na equação geral}

Para análise dos parâmetros utilizados na Equação de Fourier para estruturas de concreto massa, estudou-se um bloco de concreto de $5 \mathrm{~m}$ x $5 \mathrm{~m}$, com uma malha de 0,10 m, 2601 nós e 2500 elementos no ANSYS. O elemento utilizado foi o PLANE55 da biblioteca do ANSYS que tem capacidade de condução térmica em duas dimensões, 2D, suficientemente testado e avaliado externamente. O elemento tem quatro nós e um único grau de liberdade em cada nó, a temperatura.

As isotermas de temperatura foram analisadas após dois, quatro e seis dias de concretagem. Para todos os casos, as variáveis que correspondem a cada propriedade estão relacionadas abaixo:

- Condutividade térmica $=\mathrm{k}$;

- Densidade $=\rho$;

- Calor específico = c;

- Coeficiente de convecção = h;

- Diferença de temperatura em porcentagem $=\Delta(\%)$.

É importante ressaltar que para os parâmetros analisados admitiu-se os valores máximos e mínimos permitidos para o concreto. Sendo que estes foram aproximados para valores inteiros; para o número inteiro imediatamente inferior no caso dos valores mínimos; e para o número inteiro imediatamente superior, no caso dos valores máximos.

Outra observação importante é que para esse estudo os parâmetros foram considerados variando isoladamente, ou seja, sem a dependência dos outros parâmetros. No entanto, sabese, por exemplo, que alterando-se o traço do concreto, normalmente, haverá a alteração de todos os parâmetros e não de apenas um como está sendo adotado. 
Com o intuito de esclarecer as dependências desses parâmetros, a tabela 1 mostra a relação de dependência dos mesmos.

Tabela 1. Definição dos parâmetros.

\begin{tabular}{|c|c|c|}
\hline Parâmetro & Definiçãa & Dependência \\
\hline $\begin{array}{l}\text { Condutividade } \\
\text { térmica (k) }\end{array}$ & $\begin{array}{l}\text { Capacidade do material } \\
\text { conduzir calor. }\end{array}$ & $\begin{array}{l}\text { - Composição do concreto; } \\
\text { - Teor de umidade; } \\
\text { - Características } \\
\text { mineralógicas do } \\
\text { agregado; } \\
\text { - Densidade e temperatura } \\
\text { do concreto; } \\
\text { - Não sofre influência do } \\
\text { tipo de cimento utilizado. }\end{array}$ \\
\hline $\begin{array}{l}\text { Densidade ou massa } \\
\text { específica }(\rho)\end{array}$ & $\begin{array}{l}\text { É a relação entre a massa } \\
\text { de um corpo sobre o } \\
\text { volume que esse mesmo } \\
\text { corpo ocupa. }\end{array}$ & $\begin{array}{l}\text { - Teor de água e de finos; } \\
\text { - Agregados utilizados; } \\
\text { - Índice de vazios; } \\
\text { - Temperatura do concreto. }\end{array}$ \\
\hline Calor específico (c) & $\begin{array}{l}\text { Corresponde à } \\
\text { quantidade de calor } \\
\text { necessária para elevar em } \\
\text { um grau a temperatura de } \\
\text { uma unidade de massa. }\end{array}$ & $\begin{array}{l}\text { - } \text { Temperatura; } \\
\text { - } \text { Massa específica do } \\
\text { concreto; } \\
\text { - } \text { Teor de umidade; } \\
\text { - } \text { Dimensão máxima } \\
\text { característica do agregado } \\
\text { graúdo. }\end{array}$ \\
\hline
\end{tabular}

Para a análise foram admitidos temperatura ambiente de $20^{\circ} \mathrm{C}$, temperatura na face inferior do bloco de $25^{\circ} \mathrm{C}$ e temperatura de lançamento do concreto de $45^{\circ} \mathrm{C}$. Os resultados dos parâmetros característicos encontram-se nas figuras 2 a 7 e nas tabelas de 2 a 4 . Vale salientar que o nó de temperatura máxima está indicado como o ponto preto da figura.

\subsubsection{Condutividade térmica}

As figuras 2 e 3 e a tabela 2 sumarizam os principais resultados obtidos para dois valores (mínimo e máximo) da condutividade térmica do concreto. 


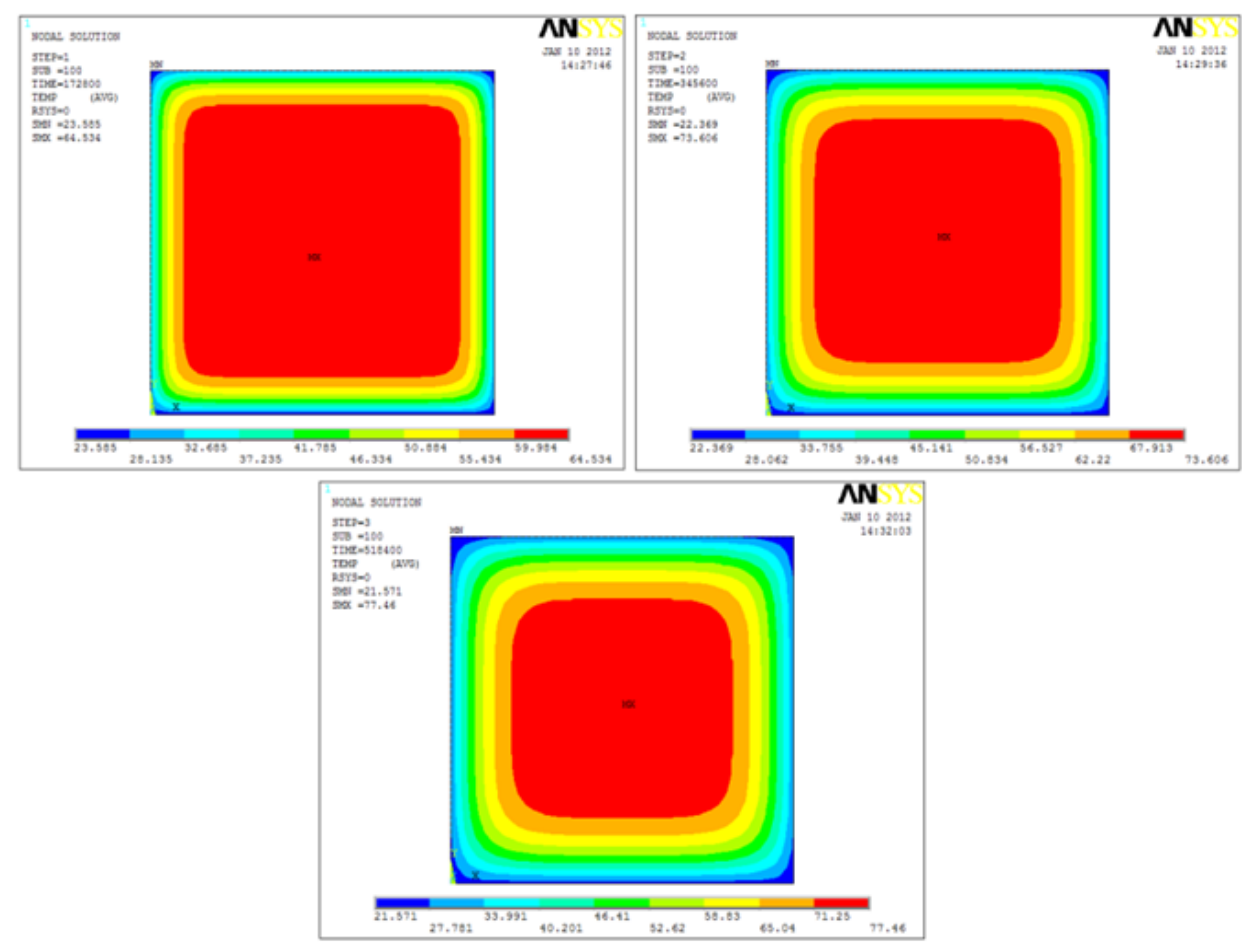

Figura 2. Isotermas de temperatura para 2,4 e 6 dias de concretagem, com $\mathrm{k}=1 \mathrm{~W} / \mathrm{m} .{ }^{\circ} \mathrm{C}$.

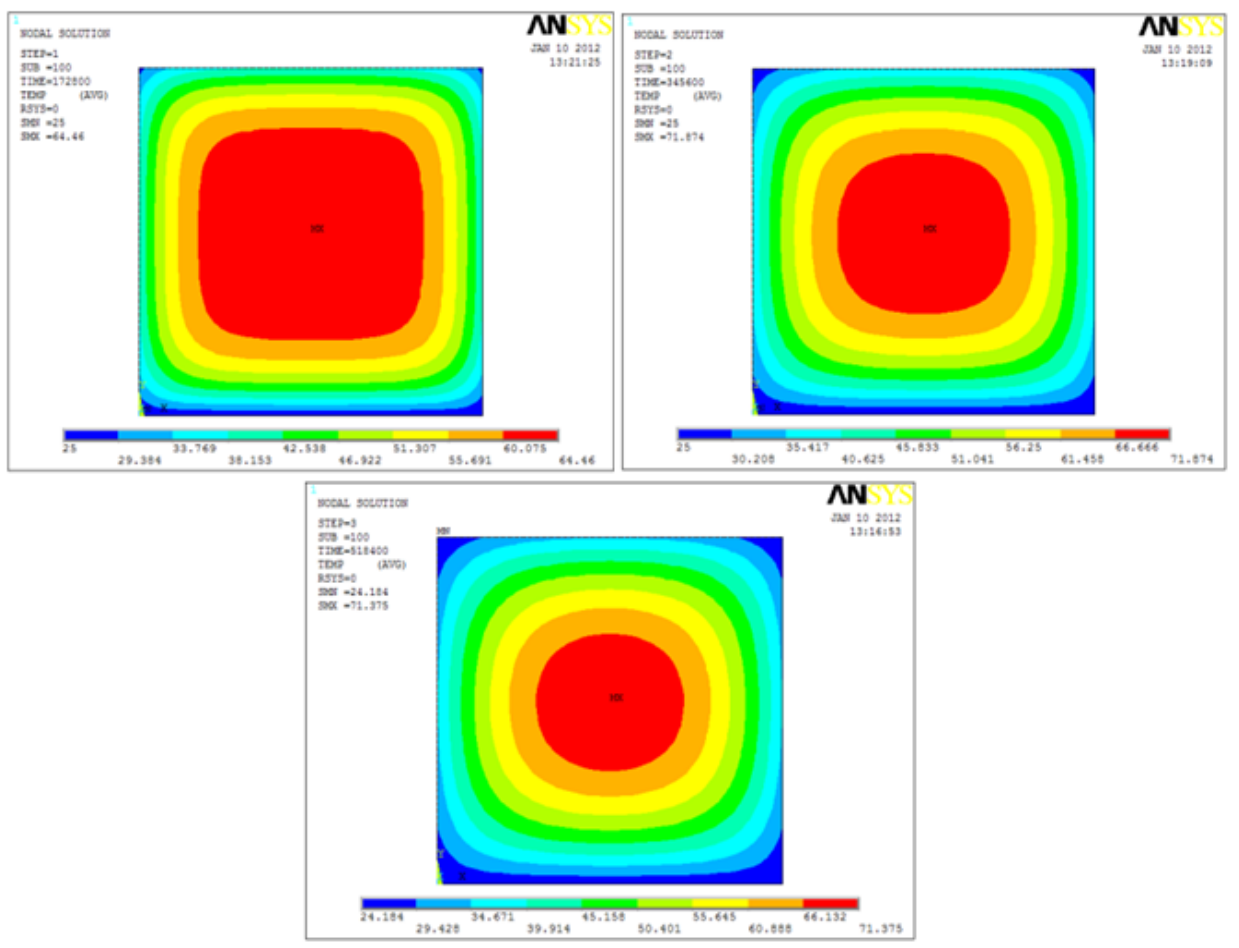

Figura 3. Isotermas de temperatura para 2,4 e 6 dias de concretagem, com $\mathrm{k}=4 \mathrm{~W} / \mathrm{m} .{ }^{\circ} \mathrm{C}$. 
Tabela 2. Resultados para diferentes valores de $\mathrm{k}$.

\begin{tabular}{|c|c|c|c|}
\hline \multirow{2}{*}{ Propriedades } & \multicolumn{3}{|c|}{ Temperatura máxima; Nó } \\
\hline & 2 dias & 4 dias & 6 dias \\
\hline $\begin{array}{l}\mathrm{k}=1 \mathrm{~W} / \mathrm{m} \cdot{ }^{\circ} \mathrm{C} \\
\rho=2300 \mathrm{~kg} / \mathrm{m}^{3} \\
\mathrm{c}=1000 \mathrm{~J} / \mathrm{g} \cdot{ }^{\circ} \mathrm{C} \\
\mathrm{h}=10 \mathrm{~W} / \mathrm{m}^{2} .{ }^{\circ} \mathrm{C}\end{array}$ & $\begin{array}{c}64.534^{\circ} \mathrm{C} \\
\text { Nó }=1401\end{array}$ & $\begin{array}{c}73.606^{\circ} \mathrm{C} \\
\text { Nó }=1401\end{array}$ & $\begin{array}{c}77.460^{\circ} \mathrm{C} \\
\text { Nó }=1401\end{array}$ \\
\hline $\begin{array}{l}\mathrm{k}=4 \mathrm{~W} / \mathrm{m} .{ }^{\circ} \mathrm{C} \\
\rho=2300 \mathrm{~kg} / \mathrm{m}^{3} \\
\mathrm{c}=1000 \mathrm{~J} / \mathrm{g} .{ }^{\circ} \mathrm{C} \\
\mathrm{h}=10 \mathrm{~W} / \mathrm{m}^{2} .{ }^{\circ} \mathrm{C}\end{array}$ & $\begin{array}{c}64.460^{\circ} \mathrm{C} \\
\text { Nó }=1402\end{array}$ & $\begin{array}{c}71.874^{\circ} \mathrm{C} \\
\text { Nó }=1402\end{array}$ & $\begin{array}{c}71.375^{\circ} \mathrm{C} \\
\text { Nó }=1402\end{array}$ \\
\hline$\Delta(\%)$ & 0,11 & 2,41 & 8,52 \\
\hline
\end{tabular}

Observa-se que quanto menor a condutividade térmica do concreto, maior será a temperatura máxima atingida. Isso pode ser explicado pelo fato de que com uma menor condutividade térmica, maior será a dificuldade de dissipação do calor gerado internamente nesse corpo. Nota-se também que o parâmetro 'tempo' influencia diretamente nestas isotermas. Com o tempo, observa-se que a região que apresenta temperatura máxima diminui.

\subsubsection{Calor específico}

As figuras 4 e 5 e a tabela 3 sintetizam os resultados obtidos para dois valoresextremos do calor específico do concreto.

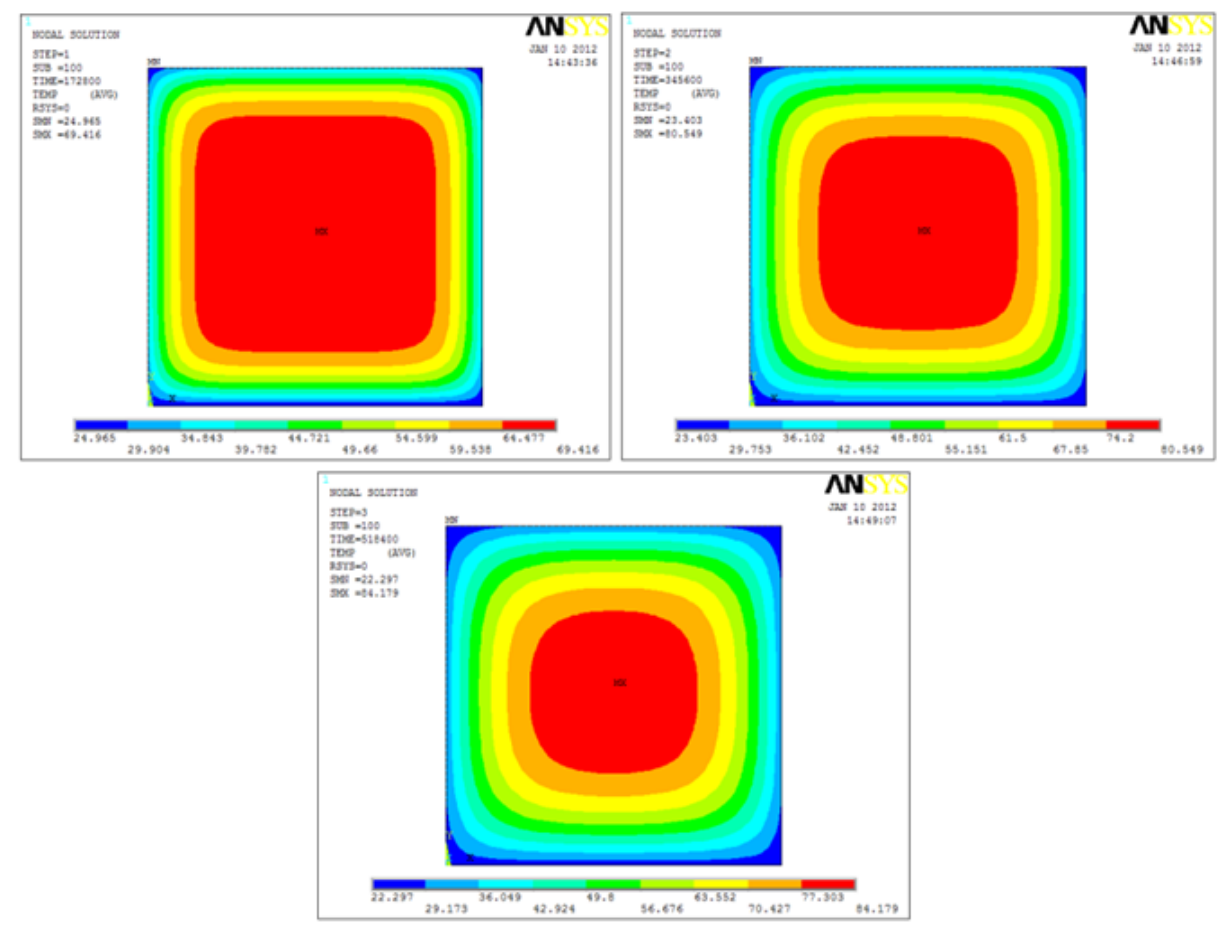

Figura 4. Isotermas de temperatura para 2, 4 e 6 dias de concretagem, com c $=800 \mathrm{~J} / \mathrm{g}^{\circ} \mathrm{C}$. 


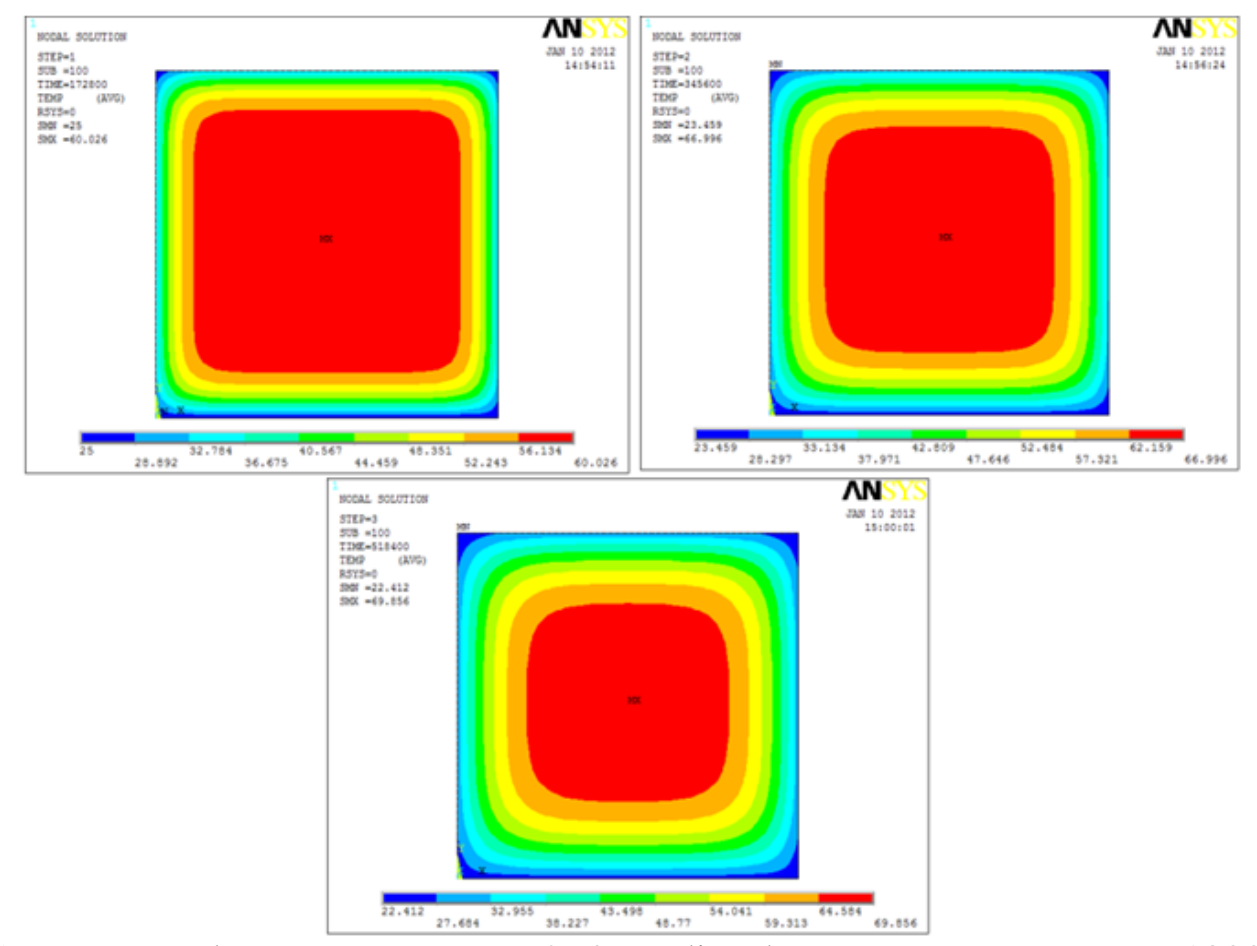

Figura 5. Isotermas de temperatura para 2, 4 e 6 dias de concretagem, com $\mathrm{c}=1300 \mathrm{~J} / \mathrm{g}^{\circ} \mathrm{C}$.

Tabela 3. Resultados para diferentes valores de c.

\begin{tabular}{|c|c|c|c|}
\hline \multirow{2}{*}{ Propriedades } & \multicolumn{3}{|c|}{ Temperatura máxima; Nó } \\
\hline & 2 dias & 4 dias & 6 dias \\
\hline \multicolumn{4}{|l|}{$\mathrm{k}=1,79 \mathrm{~W} / \mathrm{m} \cdot{ }^{\circ} \mathrm{C}$} \\
\hline$\rho=2300 \mathrm{~kg} / \mathrm{m}^{3}$ & $69.416^{\circ} \mathrm{C}$ & $80.549^{\circ} \mathrm{C}$ & $84.179^{\circ} \mathrm{C}$ \\
\hline $\mathrm{c}=800 \mathrm{~J} / \mathrm{g} \cdot{ }^{\circ} \mathrm{C}$ & Nó = 1401 & Nó = 1401 & Nó = 1402 \\
\hline \multicolumn{4}{|l|}{$\mathrm{h}=10 \mathrm{~W} / \mathrm{m}^{2}{ }^{\circ} \mathrm{C}$} \\
\hline \multicolumn{4}{|l|}{$\mathrm{k}=1,79 \mathrm{~W} / \mathrm{m} .{ }^{\circ} \mathrm{C}$} \\
\hline$\rho=2300 \mathrm{~kg} / \mathrm{m}^{3}$ & $60.026^{\circ} \mathrm{C}$ & $66.996^{\circ} \mathrm{C}$ & $69.856^{\circ} \mathrm{C}$ \\
\hline $\mathrm{c}=1300 \mathrm{~J} / \mathrm{g} \cdot{ }^{\circ} \mathrm{C}$ & Nó = 1401 & Nó = 1401 & Nó = 1401 \\
\hline \multicolumn{4}{|l|}{$\mathrm{h}=10 \mathrm{~W} / \mathrm{m}^{2}{ }^{\circ} \mathrm{C}$} \\
\hline$\Delta(\%)$ & 15,64 & 20,23 & 20,50 \\
\hline
\end{tabular}

Sabe-se que o calor específico corresponde à quantidade de calor necessária para elevar em um grau a temperatura de uma unidade de massa; e que atinge um valor máximo para o concreto saturado. Observa-se que para um menor valor de calor específico, têm-se maiores temperaturas, ou seja, uma quantidade menor de calor é necessária para que haja elevação de temperatura. No entanto, para um maior valor de calor específico, as isotermas de temperatura máxima apresentam-se maiores que para o caso com menor calor específico. 


\subsubsection{Massa Específica}

As figuras 6 e 7 e a tabela 4 mostram os resultados obtidos para dois valores diferentes (extremos) da massa específica do concreto.
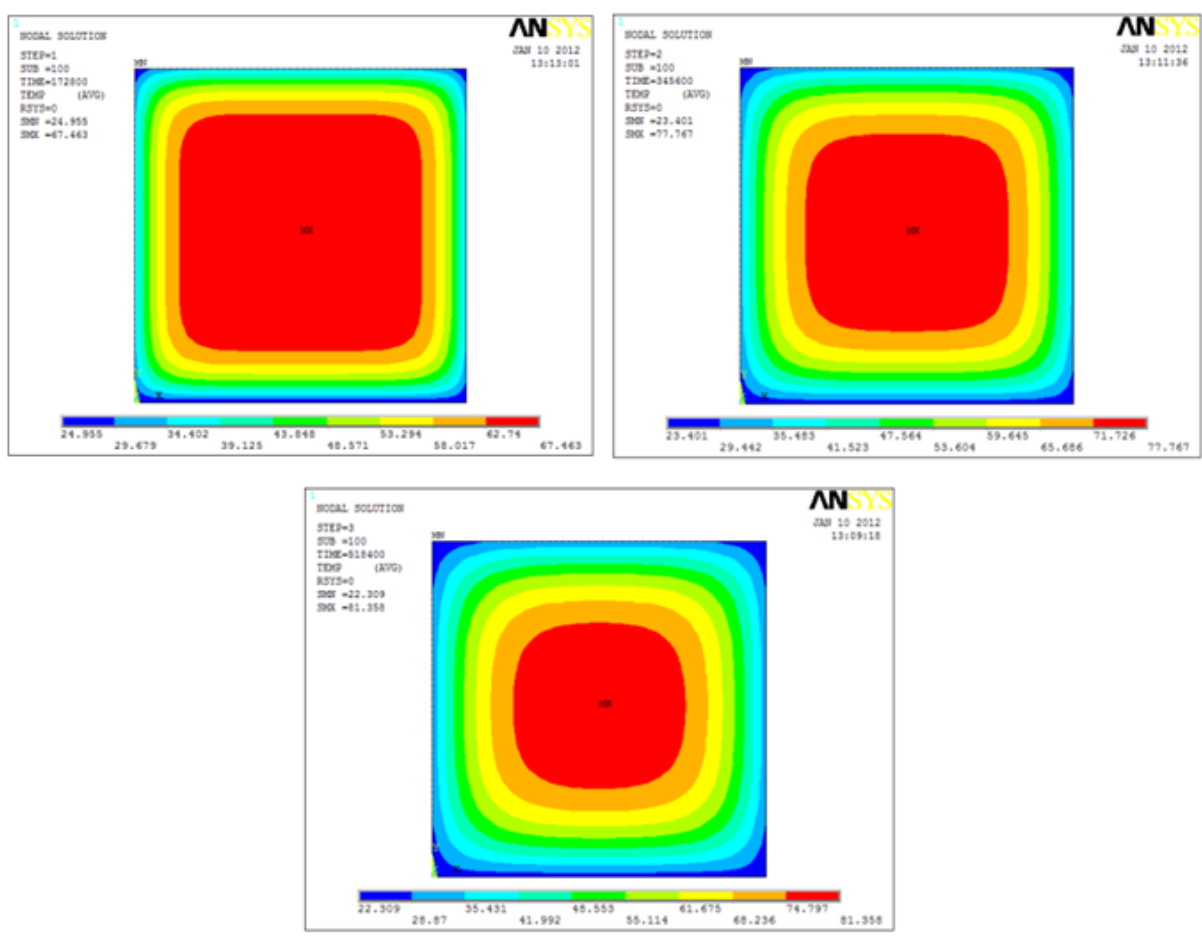

Figura 6. Isotermas de temperatura para 2, 4 e 6 dias de concretagem, com $\rho=2000 \mathrm{~kg} / \mathrm{m}^{3}$.

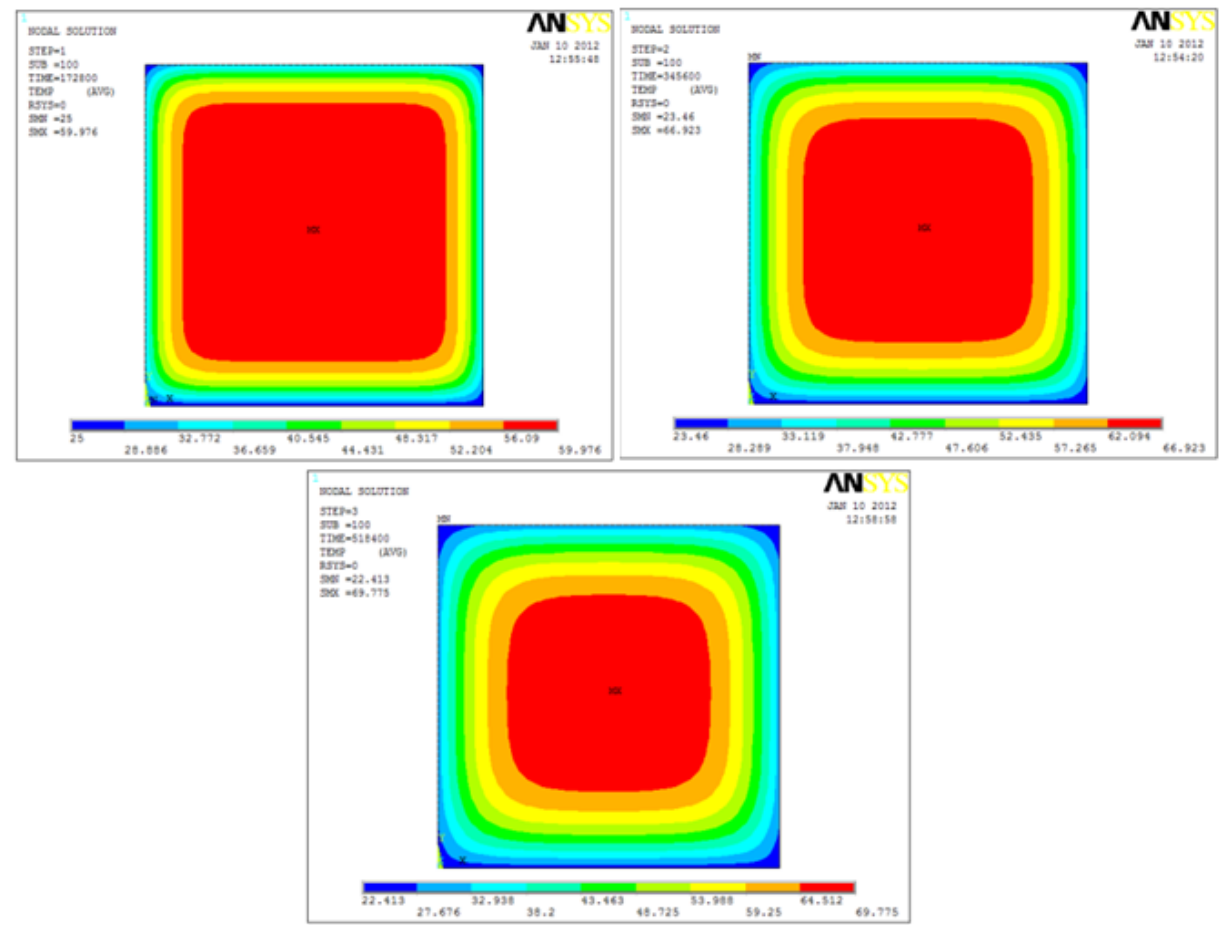

Figura 7. Isotermas de temperatura para 2, 4 e 6 dias de concretagem, com $\rho=3000 \mathrm{~kg} / \mathrm{m}^{3}$. 
Tabela 4. Resultados para diferentes valores de $\rho$.

\begin{tabular}{|c|c|c|c|}
\hline \multirow{2}{*}{ Propriedades } & \multicolumn{3}{|c|}{ Temperatura máxima; Nó } \\
\hline & 2 dias & 4 dias & 6 dias \\
\hline \multicolumn{4}{|l|}{$\mathrm{k}=1,79 \mathrm{~W} / \mathrm{m} \cdot{ }^{\circ} \mathrm{C}$} \\
\hline$\rho=2000 \mathrm{~kg} / \mathrm{m}^{3}$ & $67.463^{\circ} \mathrm{C}$ & $77.767^{\circ} \mathrm{C}$ & $81.358^{\circ} \mathrm{C}$ \\
\hline $\mathrm{c}=1000 \mathrm{~J} / \mathrm{g} \cdot{ }^{\circ} \mathrm{C}$ & Nó $=1401$ & Nó $=1401$ & Nó $=1401$ \\
\hline \multicolumn{4}{|l|}{$\mathrm{h}=10 \mathrm{~W} / \mathrm{m}^{2}{ }^{\circ} \mathrm{C}$} \\
\hline \multicolumn{4}{|l|}{$\mathrm{k}=1,79 \mathrm{~W} / \mathrm{m} \cdot{ }^{\circ} \mathrm{C}$} \\
\hline$\rho=3000 \mathrm{~kg} / \mathrm{m}^{3}$ & $59.976^{\circ} \mathrm{C}$ & $66.923^{\circ} \mathrm{C}$ & $69.775^{\circ} \mathrm{C}$ \\
\hline $\mathrm{c}=1000 \mathrm{~J} / \mathrm{g} \cdot{ }^{\circ} \mathrm{C}$ & Nó $=1401$ & Nó = 1401 & Nó $=1401$ \\
\hline \multicolumn{4}{|l|}{$\mathrm{h}=10 \mathrm{~W} / \mathrm{m}^{2}{ }^{\circ} \mathrm{C}$} \\
\hline$\Delta(\%)$ & 12,48 & 16,20 & 16,60 \\
\hline
\end{tabular}

Com a redução da massa específica tem-se o aumento de temperatura. Sabe-se que a massa específica é a relação entre a massa de um corpo sobre o volume que esse mesmo corpo ocupa; e que a redução da relação água/cimento na mistura aumenta sua massa específica. Dessa forma, pode-se afirmar que para uma menor massa específica, têm-se um maior isolamento, fato que dificulta a dissipação interna de calor.

\section{CONCLUSÕES}

Um dos fatores de mais influência nos efeitos térmicos é a geração do calor interno no concreto. Quando os grãos do cimento entram em contato com a água eles se hidratam e liberam calor. Essa geração de calor provoca as tensões térmicas que podem causar danos a estrutura. No entanto, essas tensões são mais significativas quando há um maior gradiente de temperatura, ou seja, quando há maior diferença entre as temperaturas máximas e mínimas no concreto massa.

Foram realizadas diversas análises para estruturas em concreto massa, onde os efeitos térmicos puderam ser analisados através das isotermas de temperatura obtidas com o software ANSYS. Essas isotermas indicam a evolução do calor, mostrando temperaturas no domínio do corpo estudado.

Notou-se que uma menor a condutividade térmica do concreto implica em uma maior temperatura máxima devido à dificuldade de dissipação do calor gerado internamente nesse corpo. Com a redução da massa específica tem-se o aumento da temperatura. Isso porque têmse uma maior porosidade, o que dificulta a dissipação interna de calor. Para o calor especifico, quanto menor o valor, maior a temperatura, pois uma quantidade menor de calor é necessária para que haja elevação de temperatura. Dessa forma, percebe-se que para redução do calor interno é desejável um concreto com uma maior condutividade térmica, densidade e calor específico. No entanto, essas propriedades estabelecem uma relação de dependência que não foi considerada neste trabalho. 
Verificou-se ainda que o uso de um programa baseado no método dos elementos finitos na solução dos problemas, pode ser um meio rápido e prático para uma análise térmica em estruturas. Isso possibilita que as estruturas sejam analisadas antes mesmo da construção, permitindo assim estratégias para a redução de tensões devido aos efeitos térmicos.

\section{Agradecimentos}

Agradecimentos à equipe do PECC - Programa de Pós-Graduação em Estruturas e Construção Civil da UnB - Universidade de Brasília.

\section{REFERENCIAS}

[1] American Concrete Institute. "Cement and Concrete Terminology". ACI 318 Farmington Hills, 2005.

[2] Associação Brasileira de Normas Técnicas. "NBR 12818: Concreto - Determinação da difusividade térmica”. Rio de Janeiro: 1993.

[3] AZENHA, M. A. D.; "Numerical Simulation of The Structural Behaviour of Concrete Since Its Early Ages". 379 f. Tese de Doutorado. Faculdade de Engenharia da Universidade do Porto - FEUP. Porto: 2009.

[4] CHOKTAWEEKARN, P.; TANGTERMSIRIKUL, S.; "Effect of aggregate type, casting, thickness, and curing condition on restrained strain of mass concrete". Songklanakarin J. Sci. Technol. Thailand: 2010.

[5] FARIA, E. F.; "Predição da exotermia da reação de hidratação do concreto através de modelo termo-químico e modelo de dados”. 145 f. Dissertação de Mestrado. Universidade Federal do Rio De Janeiro - COOPE/UFRJ. Rio de Janeiro: 2004.

[6] Incropera, F. P.; Dewitt, D. P.; Bergman, T. L.; Lavine, A. S. "Fundamentos de Transferência de Calor e Massa". 6 ${ }^{a}$ ed. Rio de Janeiro: LTC, 2008.

[7] Inoue, G.; "Medidas Preventivas de Controle de Temperatura que Induz Fissuração no Concreto Massa”. Boletim Técnico No 7; Escola Politécnica da Universidade de São Paulo - EPUSP; São Paulo: 1986.

[8] KAVAMURA, E. E.; "Estudo do comportamento termo-estrutural bidimensional de barragens de concreto utilizando o Método dos Elementos Finitos”. Dissertação de Mestrado. Universidade Federal do Paraná - UFPR, Curitiba: 2005.

[9] KRÜGER, D. A. V.; "Análise térmica transiente de estruturas de concreto executadas por camadas”. Dissertação de Mestrado. Universidade Federal do Paraná - UFPR, Curitiba: 
2001

[10] Liu, J.; Qiao, L; Li, P.; Dai, C.; "Laboratory Test and Numerical Simulation of TimeDependent Thermomechanical Behavior of the Three-Gorges Dam"; Journal of Materials in Civil Engineering. February, 2010.

[11] Neville, A. M. "Propriedades do concreto". 2. ed. São Paulo: Pini, 1997. 828 p.

[12] SANTOS, S. B. Uma Contribuição ao Estudo do Comportamento Termomecânico de Estruturas Maciças de Concreto. Modelagem Viscoelástica Linear e Aplicações. $287 \mathrm{f}$. Dissertação de Mestrado. Universidade Federal do Espírito Santo. Vitória: 2004. 